Bull. Korean Math. Soc. 52 (2015), No. 3, pp. 717-734

http://dx.doi.org/10.4134/BKMS.2015.52.3.717

\title{
CLASSIFICATION ON ARITHMETIC FUNCTIONS AND CORRESPONDING FREE-MOMENT $L$-FUNCTIONS
}

\author{
ILWOO CHO
}

\begin{abstract}
In this paper, we provide a classification of arithmetic functions in terms of identically-free-distributedness, determined by a fixed prime. We show then such classifications are free from the choice of primes. In particular, we obtain that the algebra $\mathfrak{A}_{p}$ of equivalence classes under the quotient on $\mathcal{A}$ by the identically-free-distributedness is isomorphic to an algebra $\mathbb{C}^{2}$, having its multiplication $(\bullet) ;\left(t_{1}, t_{2}\right) \bullet\left(s_{1}, s_{2}\right)=$ $\left(t_{1} s_{1}, t_{1} s_{2}+t_{2} s_{1}\right)$.
\end{abstract}

\section{Introduction}

In [7], the author and Gillespie established the free probabilistic model $\left(\mathcal{A}, \varphi_{x}\right)$ on the algebra $\mathcal{A}$ consisting of all arithmetic functions by constructing linear functionals $\varphi_{x}$ on it, for all positive real numbers $x$.

As a continued study of such a free probabilistic model on $\mathcal{A}$, the author considered "truncated" linear functionals $\varphi_{x<y}$, in [6]. They contain information about primes between $x$ and $y$, and induce free probabilistic data for the primes, where $x<y \in \mathbb{R}^{+}$. In particular, we showed that the free distributional data of $f \in \mathcal{A}$ is characterized by each prime.

Arithmetic functions are main objects in modern number theory. They are the functions $f$ defined from the natural numbers $\mathbb{N}$ into the complex numbers $\mathbb{C}$. Especially, they are tools for constructing (classical) Dirichlet L-functions,

$$
L_{f}(s)=\sum_{k=1}^{\infty} \frac{f(k)}{k^{s}} \text { for all } s \in \mathbb{C},
$$

on $\mathbb{C}$, for $f \in \mathcal{A}$. The entire-ness of $L$-functions is an important and interesting topic in analysis. So, they are main objects for investigating modern number theory; combinatorial number theory, L-function theory, and analytic number theory, etc (e.g., [1], [2], [11], [12], [13], [14] and [17]).

Received February 21, 2013; Revised May 21, 2013

2010 Mathematics Subject Classification. 05E15, 11G15, 11R04, 11R09, 11R47, 11R56, 46L10, 46L40, 46L53, 46L54, 47L15, 47L30, 47L55.

Key words and phrases. arithmetic functions, arithmetic algebra, linear functionals, arithmetic probability spaces, free-moment $L$-functions. 


\subsection{Background}

We concentrate on the fact the collection $\mathcal{A}$ of all arithmetic functions forms an algebra, under the usual functional addition and convolution. Recall that if $f_{1}, f_{2}$ are arithmetic functions in $\mathcal{A}$, then the convolution $f_{1} * f_{2}$ becomes an arithmetic function in $\mathcal{A}$, too, where

$$
f_{1} * f_{2}(n) \stackrel{\text { def }}{=} \sum_{d \mid n} f_{1}(d) f_{2}\left(\frac{n}{d}\right)
$$

for all $n \in \mathbb{N}$, where " $d \mid n$ " means " $d$ divides $n$," or " $n$ is divisible by $d$," for $d \in \mathbb{N}$.

In [7], by understanding $\mathcal{A}$ as an algebra, we defined linear functionals $\varphi_{x}$ on $\mathcal{A}$ by

$$
\varphi_{x}(f) \stackrel{\text { def }}{=} \sum_{p: \text { primes, } p \leq x} f(p) \text { for all } f \in \mathcal{A} .
$$

It provides corresponding free probability on $\mathcal{A}$, i.e., each element $f$ of $\mathcal{A}$ can have its distributional data in terms of $\varphi_{x}$. The existence of the system $\left\{\varphi_{x}\right\}_{x \in \mathbb{R}^{+}}$guarantees how the real numbers $\mathbb{R}$ acts on $\mathcal{A}$. We have studied freeprobabilistic properties of $\left\{\left(\mathcal{A}, \varphi_{x}\right)\right\}_{x \in \mathbb{R}^{+}}$, and considered the relations between free probabilistic data and well-known number-theoretic results in [7].

In [6], we defined a new type of linear functionals $\varphi_{x<y}$, for $x<y$ in $\mathbb{R}^{+}$;

$$
\varphi_{x<y}(f) \stackrel{\text { def }}{=} \sum_{p: \text { prime, } x<p \leq y} f(p) \text { for all } f \in \mathcal{A},
$$

and established the corresponding free probability space $\left(\mathcal{A}, \varphi_{x<y}\right)$.

We study relations between $\left(\mathcal{A}, \varphi_{x<y}\right)$ and $\left\{\left(\mathcal{A}, \varphi_{t}\right)\right\}_{t \in \mathbb{R}^{+}}$in $[6]$. We realized that every prime $p$ acts as a linear functional $g_{p}$ on $\mathcal{A}$, i.e.,

$$
g_{p}(f) \stackrel{\text { def }}{=} f(p) \text { for all } f \in \mathcal{A} \text {. }
$$

\subsection{Overview}

In this paper, based on the results of [6] and [7], we concentrate on studying (i) free moments of arithmetic functions, (ii) the identically free-distributedness conditions, and (iii) the corresponding free-moment L-functions in the sense of $[6]$.

The main results are the classification theorem of the arithmetic algebra $\mathcal{A}$, and that of the algebra $\mathcal{L}_{p}$, consisting of all free-moment $L$-functions in $\left(\mathcal{A}, g_{p}\right)$, under an equivalence relation induced by identically free-distributedness.

In Section 2, we briefly introduce free probability theory providing a main tool for our study. In Section 3, free moments of arithmetic functions are computed. From the computation, we construct a classification on $\mathcal{A}$. Corresponding free-moment $L$-functions are studied in Section 4. 


\section{Free probability}

Let $\mathfrak{A}$ be an arbitrary algebra, and let $\psi: \mathfrak{A} \rightarrow \mathbb{C}$ be a linear functional on $\mathfrak{A}$. Then the pair $(\mathfrak{A}, \psi)$ is called a free probability space (over $\mathbb{C}$ ). All operators $a \in(\mathfrak{A}, \psi)$ are called free random variables (See [16] and [18]). Remark that free probability spaces are dependent upon the choice of linear functionals.

Let $a_{1}, \ldots, a_{s}$ be a free random variable in a $(\mathfrak{A}, \psi)$, for $m \in \mathbb{N}$. The free moments of $a_{1}, \ldots, a_{m}$ are determined by the quantities

$$
\psi\left(a_{i_{1}} \cdots a_{i_{n}}\right)
$$

for all $\left(i_{1}, \ldots, i_{n}\right) \in\{1, \ldots, m\}^{n}$, for all $n \in \mathbb{N}$.

And the free cumulants $k_{n}\left(a_{i_{1}}, \ldots, a_{i_{n}}\right)$ of $a_{1}, \ldots, a_{m}$ is determined by the Möbius inversion;

$$
\begin{aligned}
k_{n}\left(a_{i_{1}}, \ldots, a_{i_{n}}\right) & =\sum_{\pi \in N C(n)} \psi_{\pi}\left(a_{i_{1}}, \ldots, a_{i_{n}}\right) \mu\left(\pi, 1_{n}\right) \\
& =\sum_{\pi \in N C(n)}\left(\prod_{V \in \pi} \psi_{V}\left(a_{i_{1}}, \ldots, a_{i_{n}}\right) \mu\left(0_{|V|}, 1_{|V|}\right)\right)
\end{aligned}
$$

for all $\left(i_{1}, \ldots, i_{n}\right) \in\{1, \ldots, m\}^{n}$, for all $n \in \mathbb{N}$, where $\psi_{\pi}(\cdots)$ means the partition-depending moments, and $\psi_{V}(\cdots)$ means the block-depending moment; for example, if

$$
\pi=\{(1,5,7),(2,3,4),(6)\} \text { in } N C(7),
$$

with three blocks $(1,5,7),(2,3,4)$, and $(6)$, then

$$
\begin{aligned}
\psi_{\pi}\left(a_{i_{1}}, \ldots, a_{i_{7}}\right) & =\psi_{(1,5,7)}\left(a_{i_{1}}, \ldots, a_{i_{7}}\right) \psi_{(2,3,4)}\left(a_{i_{1}}, \ldots, a_{i_{7}}\right) \psi_{(6)}\left(a_{i_{1}}, \ldots, a_{i_{7}}\right) \\
& =\psi\left(a_{i_{1}} a_{i_{5}} a_{i_{7}}\right) \psi\left(a_{i_{2}} a_{i_{3}} a_{i_{4}}\right) \psi\left(a_{i_{6}}\right) .
\end{aligned}
$$

Here, the set $N C(n)$ means the noncrossing partition set over $\{1, \ldots, n\}$, which is a lattice with the inclusion $\leq$, such that

$$
\theta \leq \pi \stackrel{\text { def }}{\Longleftrightarrow} \forall V \in \theta, \exists B \in \pi \text { s.t., } V \subseteq B,
$$

where $V \in \theta$ or $B \in \pi$ means that $V$ is a block of $\theta$, respectively, $B$ is a block of $\pi$, and $\subseteq$ means the usual set inclusion. This lattice has its minimal element $0_{n}=\{(1),(2), \ldots,(n)\}$, the $n$-block partition, and its maximal element $1_{n}=\{(1, \ldots, n)\}$, the one-block partition.

Especially, a partition-depending free moment $\psi_{\pi}(a, \ldots, a)$ is determined by

$$
\psi_{\pi}(a, \ldots, a)=\prod_{V \in \pi} \psi\left(a^{|V|}\right),
$$

where $|V|$ means the cardinality of $V$.

Also, $\mu$ is the Möbius functional from $\mathcal{N C}$ into $\mathbb{C}$, where

$$
\mathcal{N C}=\bigcup_{n=1}^{\infty}(N C(n) \times N C(n))
$$

i.e., it satisfies

$$
\mu(\pi, \theta)=0 \text { for all } \pi>\theta \text { in } N C(n)
$$


and

$$
\mu\left(0_{n}, 1_{n}\right)=(-1)^{n-1} c_{n-1}, \text { and } \sum_{\pi \in N C(n)} \mu\left(\pi, 1_{n}\right)=0
$$

for all $n \in \mathbb{N}$, where

$$
c_{k}=\frac{1}{k+1}\left(\begin{array}{c}
2 k \\
k
\end{array}\right)=\frac{1}{k+1} \frac{(2 k) !}{k ! k !}
$$

means the $k$-th Catalan numbers, for all $k \in \mathbb{N}$. Notice that since each $N C(n)$ is a well-defined lattice, if $\pi<\theta$ are given in $N C(n)$, one can decide the "interval"

$$
[\pi, \theta]=\{\delta \in N C(n): \pi \leq \delta \leq \theta\},
$$

and it is always lattice-isomorphic to

$$
[\pi, \theta]=N C(1)^{k_{1}} \times N C(2)^{k_{2}} \times \cdots \times N C(n)^{k_{n}}
$$

for some $k_{1}, \ldots, k_{n} \in \mathbb{N}$, where $N C(l)^{k_{t}}$ means " $l$ blocks of $\pi$ generates $k_{t}$ blocks of $\theta$," for $k_{j} \in\{0,1, \ldots, n\}$, for all $n \in \mathbb{N}$. By the multiplicativity of $\mu$ on $N C(n)$, for all $n \in \mathbb{N}$, if an interval $[\pi, \theta]$ in $N C(n)$ satisfies the above set-product relation, then we have

$$
\mu(\pi, \theta)=\prod_{j=1}^{n} \mu\left(0_{j}, 1_{j}\right)^{k_{j}} .
$$

(For details, see [16]. For applications, see [9] and [10].)

In fact, the free moments of free random variables and the free cumulants of them provide equivalent free distributional data. For example, if a free random variable $a$ in $(\mathfrak{A}, \psi)$ is a self-adjoint operator in a topological *-algebra $\mathfrak{A}$ in the sense that: $a^{*}=a$, then both free moments $\left\{\psi\left(a^{n}\right)\right\}_{n=1}^{\infty}$ and free cumulants $\left\{k_{n}(a, \ldots, a)\right\}_{n=1}^{\infty}$ give its spectral distributional data.

However, their uses are different case-by-case. For instance, to study the free distribution of fixed free random variables, the computation and investigation of free moments is better; and to study (inner) free structures of free random variables in $\mathfrak{A}$, the computation and observation of free cumulants is better (See $[15]$ and $[16])$.

We say two subalgebras $A_{1}$ and $A_{2}$ of $\mathfrak{A}$ are free in $(\mathfrak{A}, \psi)$, if all "mixed" free cumulants of $A_{1}$ and $A_{2}$ vanish. Two subsets $X_{1}$ and $X_{2}$ are free in $(\mathfrak{A}, \psi)$, if the subalgebras $A_{1}$ and $A_{2}$ generated by $X_{1}$ respectively $X_{2}$ are free in $(\mathfrak{A}, \psi)$. Similarly, two free random variables $a_{1}$ and $a_{2}$ are free in $(\mathfrak{A}, \psi)$, if two subsets $\left\{a_{1}\right\}$ and $\left\{a_{2}\right\}$ are free in $(\mathfrak{A}, \psi)$.

Assume that $\left\{A_{i}\right\}_{i \in \Lambda}$ is a family of subalgebras of $\mathfrak{A}$, generating $\mathfrak{A}$, i.e., $\mathfrak{A}$ is generated by $\left\{A_{i}\right\}_{i \in \Lambda}$. If $A_{i}$ 's are free from each other in $(\mathfrak{A}, \psi)$, then we say $\mathfrak{A}$ is a free product algebra with its free blocks $\left\{A_{i}\right\}_{i \in \Lambda}$, and denote this relation by

$$
\mathfrak{A}=\underset{i \in \Lambda}{* \mathbb{C}} A_{i} .
$$




\section{Free moments of arithmetic functions determined by primes}

Define

$$
\mathcal{A} \stackrel{\text { def }}{=}\{f: \mathbb{N} \rightarrow \mathbb{C}: f \text { is a function }\},
$$

i.e., $\mathcal{A}$ is the set of all arithmetic functions. Then under the usual functional addition, if $t_{1}, t_{2} \in \mathbb{C}$, and $f_{1}, f_{2} \in \mathcal{A}$, then

$$
t_{1} f_{1}+t_{2} f_{2} \in \mathcal{A} \text {. }
$$

Thus, the set $\mathcal{A}$ is a vector space over $\mathbb{C}$. Consider now the convolution $(*)$ on $\mathcal{A}$,

$$
f_{1} * f_{2}(n) \stackrel{\text { def }}{=} \sum_{d \mid n} f_{1}(d) f_{2}\left(\frac{n}{d}\right) \quad \text { for all } n \in \mathbb{N} .
$$

Then $f_{1} * f_{2}$ is an arithmetic function in $\mathcal{A}$, too.

Since this convolution is associative,

$$
\left(f_{1} * f_{2}\right) * f_{3}=f_{1} *\left(f_{2} * f_{3}\right),
$$

and since it is distributed,

$$
\begin{aligned}
& f_{1} *\left(f_{2}+f_{3}\right)=f_{1} * f_{2}+f_{1} * f_{3}, \\
& \left(f_{1}+f_{2}\right) * f_{3}=f_{1} * f_{3}+f_{2} * f_{3}
\end{aligned}
$$

for all $f_{1}, f_{2}, f_{3} \in \mathcal{A}$, the vector space $\mathcal{A}$ becomes an algebra equipped with $(*)$.

Definition 3.1. We call $\mathcal{A}$ the arithmetic(-functional) algebra.

Remark that

$$
f_{1} * f_{2}=f_{2} * f_{1} \text { for all } f_{1}, f_{2} \in \mathcal{A} .
$$

So, the arithmetic algebra $\mathcal{A}$ is a commutative algebra.

Define a linear functional $g_{p}$ on $\mathcal{A}$ for a fixed prime $p$ by

$$
g_{p}(f) \stackrel{\text { def }}{=} f(p) \text { for all } f \in \mathcal{A} \text {. }
$$

Definition 3.2. Let $\mathcal{A}$ be the arithmetic algebra, and let $g_{p}$ be the linear functional in the sense of (3.1) for a fixed prime $p$. Then the free probability space $\left(\mathcal{A}, g_{p}\right)$ is called the arithmetic $p$-prime probability space of $\mathcal{A}$.

In [6], we obtained the following free moment computation needed for our study.

Proposition 3.1 (See $[6])$. Let $\left(\mathcal{A}, g_{p}\right)$ be the arithmetic p-prime-probability space, for a fixed prime $p$. If $f$ is a free random variable in $\left(\mathcal{A}, g_{p}\right)$, then

$$
g_{p}\left(f^{(n)}\right)=n f(1)^{n-1} f(p) \text { for all } n \in \mathbb{N},
$$

where $f^{(n)}=f * \cdots * f(n$-copies $)$. 
The formula (3.2) is proven inductively, by the general relation (3.3) below,

$$
g_{p}\left(f_{1} * f_{2}\right)=g_{p}\left(f_{1}\right) f_{2}(1)+f_{1}(1) g_{p}\left(f_{2}\right)
$$

for all $f_{1}, f_{2} \in \mathcal{A}$, for all primes $p$ (See $[6]$ ).

Recall that two free random variables $a_{j}$ of free probability spaces $\left(\mathfrak{A}_{j}, \psi_{j}\right)$, for $j=1,2$, are said to be identically (free-)distributed, if

$$
\psi_{1}\left(a_{1}^{n}\right)=\psi_{2}\left(a_{2}^{n}\right) \text { for all } n \in \mathbb{N},
$$

where $\mathfrak{A}_{j}$ are (pure-algebraic) algebras and $\psi_{j}$ are linear functionals on $A_{j}$, for $j=1,2$.

By (3.2) and (3.3), one obtains the following identically distributedness conditions.

Theorem 3.2. Let $f_{1}$ and $f_{2}$ be arithmetic functions in the arithmetic algebra $\mathcal{A}$. Suppose $f_{j} \in\left(\mathcal{A}, g_{p_{j}}\right)$ for $j=1,2$. Then $f_{1}$ and $f_{2}$ are identically distributed if and only if

$$
f_{1}(1)=f_{2}(1) \text {, and } f_{1}\left(p_{1}\right)=f_{2}\left(p_{2}\right) \text { in } \mathbb{C} \text {. }
$$

Proof. $(\Leftarrow)$ Assume that

$$
f_{1}(1)=w=f_{2}(1) \text { and } f_{1}\left(p_{1}\right)=u=f_{2}\left(p_{2}\right) \text { in } \mathbb{C}
$$

for $f_{j} \in\left(\mathcal{A}, g_{p_{j}}\right)$, for $j=1,2$. Then

$$
g_{p_{1}}\left(f_{1}^{(n)}\right)=n f_{1}(1)^{n-1} f_{1}\left(p_{1}\right)=n w^{n-1} u,
$$

and

$$
g_{p_{2}}\left(f_{2}^{(n)}\right)=n f_{2}(1)^{n-1} f_{2}\left(p_{2}\right)=n w^{n-1} u
$$

for all $n \in \mathbb{N}$, by (3.2). Therefore, the free random variables $f_{j} \in\left(\mathcal{A}, g_{p_{j}}\right)$ are identically distributed from each other, for $j=1,2$.

$(\Rightarrow)$ Suppose $f_{j}$ are free random variables in the arithmetic $p_{j}$-prime probability spaces $\left(\mathcal{A}, g_{p_{j}}\right)$, for $j=1,2$. And assume that they are identically distributed, i.e.,

$$
\begin{aligned}
g_{p_{1}}\left(f_{1}^{(n)}\right) & =n f_{1}(1)^{n-1} f_{1}\left(p_{1}\right) \\
& =n f_{2}(1)^{n-1} f_{2}\left(p_{2}\right)=g_{p_{2}}\left(f_{2}^{(n)}\right)
\end{aligned}
$$

for all $n \in \mathbb{N}$.

Assume now that either $f_{1}(1) \neq f_{2}(1)$, or $f_{1}(p) \neq f_{2}(p)$ in $\mathbb{C}$.

First, assume $f_{1}(p) \neq f_{2}(p)$ in $\mathbb{C}$. Then the first free moments satisfy that

$$
g_{p_{1}}\left(f_{1}\right)=f_{1}\left(p_{1}\right) \neq f_{2}(p)=g_{p_{2}}\left(f_{2}\right),
$$

and hence, $f_{1}$ and $f_{2}$ are not identically distributed. This contradicts our assumption that $f_{1}$ and $f_{2}$ are identically distributed.

Suppose that $f_{1}\left(p_{1}\right)=d=f_{2}\left(p_{2}\right)$ in $\mathbb{C}$, and assume $f_{1}(1) \neq f_{2}(1)$ in $\mathbb{C}$. Then

$$
g_{p_{1}}\left(f_{1}^{(2)}\right)=2 f_{1}(1) f_{1}\left(p_{1}\right)=2 f_{1}(1) d
$$




$$
\neq 2 f_{2}(1) d=2 f_{2}(1) f_{2}\left(p_{2}\right)=g_{p_{2}}\left(f_{2}^{(2)}\right),
$$

by (3.3). Therefore, $f_{1}$ and $f_{2}$ are not identically distributed. It contradicts our assumption.

Finally, suppose that $f_{1}(1) \neq f_{2}(1)$ and $f_{1}\left(p_{1}\right) \neq f_{2}\left(p_{2}\right)$ in $\mathbb{C}$. Then

$$
g_{p_{1}}\left(f_{1}\right)=f_{1}\left(p_{1}\right) \neq f_{2}\left(p_{2}\right)=g_{p_{2}}\left(f_{2}\right),
$$

and hence, $f_{1}$ and $f_{2}$ are not identically distributed. It also contradicts our assumption.

Therefore, if $f_{1}(1)=f_{2}(1)$ and $f_{1}\left(p_{1}\right)=f_{2}(p)$, then $f_{1} \in\left(\mathcal{A}, g_{p_{1}}\right)$ and $f_{2} \in\left(\mathcal{A}, g_{p_{2}}\right)$ are identically distributed.

The above theorem characterizes the identically free-distributedness of arithmetic functions in terms of certain quantities.

Corollary 3.3. Let $\left(\mathcal{A}, g_{p}\right)$ be the arithmetic p-prime probability space and let $f_{1}, f_{2} \in\left(\mathcal{A}, g_{p}\right)$. Then two free random variables $f_{1}$ and $f_{2}$ are identically distributed if and only if

$$
f_{1}(1)=f_{2}(1) \text { and } f_{1}(p)=f_{2}(p) \text { in } \mathbb{C} .
$$

Also, the above characterization (3.5) provides an equivalence relation on a fixed arithmetic $p$-prime probability space $\left(\mathcal{A}, g_{p}\right)$.

Let $\left(\mathcal{A}, g_{p}\right)$ be a fixed arithmetic $p$-prime probability space for a prime $p$. Define a relation $\mathcal{R}_{p}$ on $\left(\mathcal{A}, g_{p}\right)$ by

$$
f_{1} \mathcal{R}_{p} f_{2} \stackrel{\text { def }}{\Longleftrightarrow} f_{1} \text { and } f_{2} \text { are identically distributed }
$$

in $\left(\mathcal{A}, g_{p}\right)$. Then the relation $\mathcal{R}_{p}$ is an equivalence relation. Indeed,

(i) $f \mathcal{R}_{p} f$ for all $f \in\left(\mathcal{A}, g_{p}\right)$,

(ii) $f_{1} \mathcal{R}_{p} f_{2} \Longrightarrow f_{2} \mathcal{R}_{p} f_{1}$ for all $f_{1}, f_{2} \in\left(\mathcal{A}, g_{p}\right)$, and

(iii) $f_{1} \mathcal{R}_{p} f_{2}$ and $f_{2} \mathcal{R}_{p} f_{3} \Longrightarrow f_{1} \mathcal{R}_{p} f_{3}$

for all $f_{1}, f_{2}, f_{3} \in\left(\mathcal{A}, g_{p}\right)$, by (3.4) and (3.5).

So, one can get equivalence classes $[f]_{p}$ of $f \in \mathcal{A}$ in $\left(\mathcal{A}, g_{p}\right)$, by

$$
\begin{aligned}
{[f]_{p} } & =\left\{h \in\left(\mathcal{A}, g_{p}\right): h \mathcal{R}_{p} f\right\} \\
& =\left\{h \in\left(\mathcal{A}, g_{p}\right): g_{p}\left(h^{n}\right)=g_{p}\left(f^{n}\right), \forall n \in \mathbb{N}\right\} .
\end{aligned}
$$

By (3.4) and (3.5), one obtains the following lemma.

Lemma 3.4. Let $\left(\mathcal{A}, g_{p}\right)$ be the arithmetic p-prime probability space for a prime p. Then

$$
[f]_{p}=\left\{h \in\left(\mathcal{A}, g_{p}\right): h(1)=f(1) \text { and } h(p)=f(p)\right\}
$$

for all $f \in\left(\mathcal{A}, g_{p}\right)$.

Proof. The proof of (3.8) is trivial by (3.5) and (3.7). 
The above relation (3.8) provides a classification of $\mathcal{A}$, by identically distributedness on the arithmetic algebra $\mathcal{A}$, in terms of primes, i.e., without considering all free-moments $g_{p}\left(f^{(n)}\right)$ of $f \in \mathcal{A}$, by investigating two quantities $f(1)$ and $f(p)$, one can characterize the identically distributedness in $\mathcal{A}$, in terms of primes $p$.

Theorem 3.5. Let $\left(\mathcal{A}, g_{p}\right)$ be the arithmetic p-prime probability space for a fixed prime $p$. Then

$$
\mathcal{A}=\underset{(\alpha, \lambda) \in \mathbb{C}^{2}}{\sqcup}[\alpha, \lambda]_{p}
$$

where

$$
[\alpha, \lambda]_{p} \stackrel{\text { def }}{=}\{f \in \mathcal{A}: f(1)=\alpha, f(p)=\lambda\}
$$

for all $(\alpha, \lambda) \in \mathbb{C}^{2}=\mathbb{C} \times \mathbb{C}$. Equivalently,

$$
\mathcal{A} / \mathcal{R}_{p}=\mathbb{C}^{2} \text { for all primes } p,
$$

set-theoretically.

Proof. By (3.8), each $\mathcal{R}_{p}$-equivalence class has its form,

$$
[f]_{p}=\{h \in \mathcal{A}: h(1)=f(1) \text { and } h(p)=f(p)\}
$$

for all $f \in\left(\mathcal{A}, g_{p}\right)$. So, one can realize that the equivalence classes $[f]_{p}$ are completely determined by two $\mathbb{C}$-quantities $f(1)$ and $f(p)$, i.e., every $\mathcal{R}_{p}$-equivalence class $[f]_{p}$ is determined by $\alpha=f(1)$ and $\lambda=f(p)$. So, we can re-write $[f]_{p}$ by

$$
[f(1), f(p)]_{p}=[\alpha, \lambda]_{p}
$$

as in (3.9). Since

$$
\mathcal{A}=\cup_{f \in \mathcal{A}}[f]_{p}=\underset{(f(1), f(p)) \in \mathbb{C}^{2}}{\bigcup}[f(1), f(p)]_{p}
$$

by $(3.11)$

$$
\mathcal{A}=\underset{(\alpha, \lambda) \in \mathbb{C}^{2}}{\sqcup}[\alpha, \lambda]_{p}
$$

by the uniqueness of $\mathbb{C}$-pairs in $\mathbb{C}^{2}$.

Therefore, the quotient set $\mathcal{A} / \mathcal{R}_{p}$ is equipotent (or bijective) to $\mathbb{C}^{2}$, settheoretically.

Observe now that the quotient set $\mathfrak{A}_{p}=\mathcal{A} / \mathcal{R}_{p}$ becomes an algebra, i.e., one can define

$$
\left[f_{1}\right]_{p}+\left[f_{2}\right]_{p} \stackrel{\text { def }}{=}\left[f_{1}+f_{2}\right]_{p}
$$

and

$$
\left(\left[f_{1}\right]_{p}\right)\left(\left[f_{2}\right]_{p}\right) \stackrel{\text { def }}{=}\left[f_{1} * f_{2}\right]_{p}
$$

on $\mathfrak{A}_{p}$, for all $f_{1}, f_{2} \in\left(\mathcal{A}, g_{p}\right)$.

Indeed, one can get that, for any $t_{1}, t_{2} \in \mathbb{C}$,

$$
t_{1}\left[f_{1}\right]_{p}+t_{2}\left[f_{2}\right]_{p}=\left[t_{1} f_{1}+t_{2} f_{2}\right]_{p},
$$


and hence, $\mathfrak{A}_{p}$ is a vector space over $\mathbb{C}$, furthermore,

$$
\left(\left(\left[f_{1}\right]_{p}\right)\left(\left[f_{2}\right]_{p}\right)\right)\left(\left[f_{3}\right]_{p}\right)=\left(\left[f_{1}\right]_{p}\right)\left(\left(\left[f_{2}\right]_{p}\right)\left(\left[f_{3}\right]_{p}\right)\right),
$$

in $\mathfrak{A}_{p}$, for all $f_{1}, f_{2}, f_{3} \in\left(\mathcal{A}, g_{p}\right)$, because

$$
\left(f_{1} * f_{2}\right) * f_{3}=f_{1} *\left(f_{2} * f_{3}\right) \text { on } \mathcal{A} \text {. }
$$

Furthermore, the operations (3.12) and (3.13) satisfy the distributedness,

$$
\begin{aligned}
\left(\left[f_{1}\right]_{p}\right)\left(\left[f_{2}\right]_{p}+\left[f_{3}\right]_{p}\right) & =\left(\left[f_{1}\right]_{p}\right)\left(\left[f_{2}+f_{3}\right]_{p}\right) \\
& =\left(\left[f_{1} *\left(f_{2}+f_{3}\right)\right]_{p}\right) \\
& =\left(\left[f_{1} * f_{2}+f_{1} * f_{3}\right]_{p}\right) \\
& =\left[f_{1} * f_{2}\right]_{p}+\left[f_{1} * f_{3}\right]_{p} \\
& =\left(\left[f_{1}\right]_{p}\right)\left(\left[f_{2}\right]_{p}\right)+\left(\left[f_{1}\right]_{p}\right)\left(\left[f_{3}\right]_{p}\right),
\end{aligned}
$$

similarly,

$$
\left(\left[f_{1}\right]_{p}+\left[f_{2}\right]_{p}\right)\left(\left[f_{3}\right]_{p}\right)=\left(\left[f_{1}\right]_{p}\right)\left(\left[f_{3}\right]_{p}\right)+\left(\left[f_{2}\right]_{p}\right)\left(\left[f_{3}\right]_{p}\right)
$$

for all $f_{1}, f_{2}, f_{3} \in \mathcal{A}$. Therefore, one can understand the quotient set $\mathfrak{A}_{p}=$ $\mathcal{A} / \mathcal{R}_{p}$ as an algebra.

Lemma 3.6. Let $\mathfrak{A}_{p}$ be the quotient set $\mathcal{A} / \mathcal{R}_{p}$, where $\mathcal{R}_{p}$ is the equivalence relation (3.6) on the arithmetic algebra $\mathcal{A}$. Then $\mathfrak{A}_{p}$ is an algebra over $\mathbb{C}$ with (3.12) and (3.13).

Now, let $f_{j} \in\left(\mathcal{A}, g_{p}\right)$, with

$$
f_{j}(1)=\alpha_{j}, \text { and } f_{j}(p)=\lambda_{j}
$$

for $j=1,2$. Then

and

$$
\left(f_{1}+f_{2}\right)(1)=\alpha_{1}+\alpha_{2},
$$

$$
\left(f_{1}+f_{2}\right)(p)=\lambda_{1}+\lambda_{2}
$$

Under the same hypothesis,

$$
f_{1} * f_{2}(1)=\left(f_{1}(1)\right)\left(f_{2}(1)\right)=\alpha_{1} \alpha_{2}
$$

and

$$
\begin{aligned}
\left(f_{1} * f_{2}\right)(p) & =f_{1}(1) f_{2}(p)+f_{1}(p) f_{2}(1) \\
& =\alpha_{1} \lambda_{2}+\alpha_{2} \lambda_{1},
\end{aligned}
$$

by (3.3). So, if $f_{1}$ and $f_{2}$ are given as above, then, by using the equivalent notations in $(3.9)$, one has

$$
\begin{aligned}
{\left[f_{1}\right]_{p}+\left[f_{2}\right]_{p} } & =\left[f_{1}+f_{2}\right]_{p} \\
& =\left[\alpha_{1}+\alpha_{2}, \lambda_{1}+\lambda_{2}\right]_{p}
\end{aligned}
$$

and

$$
\begin{aligned}
\left(\left[f_{1}\right]_{p}\right)\left(\left[f_{2}\right]_{p}\right) & =\left[f_{1} * f_{2}\right]_{p} \\
& =\left[\alpha_{1} \alpha_{2}, \alpha_{1} \lambda_{2}+\alpha_{2} \lambda_{1}\right]_{p} .
\end{aligned}
$$


Define now binary operations $(+)$ and $(\bullet)$ on

$$
\mathbb{C}^{2}=\{(\alpha, \lambda): \alpha, \lambda \in \mathbb{C}\}
$$

by

$$
\left(\alpha_{1}, \lambda_{1}\right)+\left(\alpha_{2}, \lambda_{2}\right) \stackrel{\text { def }}{=}\left(\alpha_{1}+\alpha_{2}, \lambda_{1}+\lambda_{2}\right),
$$

respectively,

$$
\left(\alpha_{1}, \lambda_{1}\right) \bullet\left(\alpha_{2}, \lambda_{2}\right) \stackrel{\text { def }}{=}\left(\alpha_{1} \alpha_{2}, \alpha_{1} \lambda_{2}+\alpha_{2} \lambda_{1}\right)
$$

for all $\alpha_{j}, \lambda_{j} \in \mathbb{C}$, for $j=1,2$.

It is not difficult to check that the set $\mathbb{C}^{2}$, equipped with $(+)$ and $(\bullet)$, is an algebra over $\mathbb{C}$, because for any $(\alpha, \lambda) \in \mathbb{C}^{2}$, there always exists at least one $f \in \mathcal{A}$, such that

$$
(\alpha, \lambda)=[f]_{p} .
$$

Notation. We denote this algebra $\mathbb{C}^{2}$ with (3.16) and (3.17) by $\mathfrak{C}^{2}$.

Then, we obtain the following classification theorem.

Theorem 3.7. Let $\mathfrak{A}_{p}$ be the quotient algebra $\mathcal{A} / \mathcal{R}_{p}$ with (3.12) and (3.13), and let $\mathfrak{C}^{2}$ be the algebra $\mathbb{C}^{2}$ equipped with (3.16) and (3.17). Then

$$
\mathfrak{A}_{p} \stackrel{\text { algebra }}{=} \mathfrak{C}^{2},
$$

where "algebra" means "being algebra-isomorphic," for all primes $p$.

Proof. Set-theoretically, two algebras $\mathfrak{A}_{p}$ and $\mathfrak{C}^{2}$ are equipotent, by (3.10). Define a bijective morphism

$$
\varphi: \mathfrak{C}^{2} \rightarrow \mathfrak{A}_{p}
$$

by

$$
\varphi((\alpha, \lambda))=[f]_{p}
$$

where $f$ is a free random variable of $\left(\mathcal{A}, g_{p}\right)$, such that

$$
f(1)=\alpha \text {, and } g_{p}(f)=f(p)=\lambda \text {. }
$$

Again, by (3.10), $\varphi$ is bijective under the quotient determined by the equivalence relation $\mathcal{R}_{p}$ of (3.6).

This morphism $\varphi$ satisfies

$$
\begin{aligned}
& \varphi\left(t_{1}\left(\alpha_{1}, \lambda_{1}\right)+t_{2}\left(\alpha_{2}, \lambda_{2}\right)\right) \\
= & \varphi\left(\left(t_{1} \alpha_{1}, t_{1} \lambda_{1}\right)+\left(t_{2} \alpha_{2}, t_{2} \lambda_{2}\right)\right) \\
= & \varphi\left(\left(t_{1} \alpha_{1}+t_{2} \alpha_{2}, t_{1} \lambda_{1}+t_{2} \lambda_{2}\right)\right) \\
= & {\left[t_{1} f_{1}+t_{2} f_{2}\right]_{p}\left(\text { with } f_{j}(1)=\alpha_{j}, \text { and } f_{j}(p)=\lambda_{j} \text { for } j=1,2,\right) } \\
= & t_{1}\left[f_{1}\right]_{p}+t_{2}\left[f_{2}\right]_{p}(\text { by }(3.14)) \\
= & t_{1} \varphi\left(\left(\alpha_{1}, \lambda_{1}\right)\right)+t_{2} \varphi\left(\left(\alpha_{2}, \lambda_{2}\right)\right)
\end{aligned}
$$

in $\mathfrak{A}_{p}$, for all $t_{j} \in \mathbb{C}$, and $\left(\alpha_{j}, \lambda_{j}\right) \in \mathfrak{C}^{2}$, for $j=1,2$. So, the morphism $\varphi$ is a bijective linear transformation (i.e., a vector-space isomorphism). 
Also, one can get

$$
\begin{aligned}
\varphi\left(\left(\alpha_{1}, \lambda_{1}\right) \bullet\left(\alpha_{2}, \lambda_{2}\right)\right) & =\varphi\left(\left(\alpha_{1} \alpha_{2}, \alpha_{1} \lambda_{2}+\alpha_{2} \lambda_{1}\right)\right) \\
& =\left[f_{1} * f_{2}\right]_{p}
\end{aligned}
$$

by $(3.15)$, and by the bijectivity of $\varphi$, where

$$
f_{j}(1)=\alpha_{j}, \text { and } f_{j}(p)=\lambda_{j} \text { for } j=1,2,
$$

and hence,

$$
\varphi\left(\left(\alpha_{1}, \lambda_{1}\right) \bullet\left(\alpha_{2}, \lambda_{2}\right)\right)=\left(\left[f_{1}\right]_{p}\right)\left(\left[f_{2}\right]_{p}\right)=\left(\varphi\left(\left(\alpha_{1}, \lambda_{1}\right)\right)\right)\left(\varphi\left(\left(\alpha_{2}, \lambda_{2}\right)\right)\right),
$$

in $\mathfrak{A}_{p}$, for all $\left(\alpha_{j}, \lambda_{j}\right) \in \mathfrak{C}^{2}$, for $j=1,2$. Thus the bijective linear transformation $\varphi$ is an algebra-homomorphism.

Therefore, $\varphi$ is an algebra-isomorphism, and hence, two algebras $\mathfrak{A}_{p}$ and $\mathfrak{C}^{2}$ are algebra-isomorphic, for all primes $p$.

The above classification theorem is interesting not only because it provides an isomorphism theorem of $\mathfrak{A}_{p}=\mathcal{A} / \mathcal{R}_{p}$, but also it is satisfied for all primes.

Therefore, we obtain a following isomorphism theorem, under the identicallydistributedness.

Corollary 3.8. Let $\mathfrak{A}_{p}=\mathcal{A} / \mathcal{R}_{p}$, and $\mathfrak{A}_{q}=\mathcal{A} / \mathcal{R}_{q}$, for primes $p$ and $q$, where $\mathcal{R}_{p}$ and $\mathcal{R}_{q}$ are in the sense of (3.6). Then

$$
\mathfrak{A}_{p} \stackrel{\text { Algebra }}{=} \mathfrak{A}_{q} .
$$

Proof. By (3.18), we have

$$
\mathfrak{A}_{p} \stackrel{\text { Algebra }}{=} \mathfrak{C}^{2} \stackrel{\text { Algebra }}{=} \mathfrak{A}_{q}
$$

\section{Free-moment $\boldsymbol{L}$-functions induced by primes}

In this section, we construct certain $L$-functions induced by arithmetic functions and primes. As in Section 3 , let $\left(\mathcal{A}, g_{p}\right)$ be the arithmetic $p$-prime probability space for a fixed prime $p$.

In [8], the author and Gillespie introduced free-moment L-functions induced by a fixed linear functional on an algebra. If $(A, \varphi)$ is an arbitrary free probability space, consisting of an algebra $A$ (or a normed algebra, or a *-algebra, or a $C^{*}$-algebra, or a von Neumann algebra, etc), and a linear functional $\varphi$ (resp., a continuous linear functional, resp., a linear functional with the $(*)$ property: $\varphi\left(a^{*}\right)=\overline{\varphi(a)}$, for all $a \in A$, resp., a $C^{*}$-topology continuous linear functional with $(*)$-property, resp., a $W^{*}$-topology continuous linear functional with $(*)$-property, etc), then one can define the free-moment L-function in $(A, \varphi)$

$$
L: A \times \mathbb{C} \rightarrow \mathbb{C}
$$


by

$$
L(a, s) \stackrel{\text { def }}{=} \sum_{n=1}^{\infty} \frac{\varphi\left(a^{n}\right)}{n^{s}}
$$

for all $a \in A$, and $s \in \mathbb{C}$. In particular, in [8], we considered a special case where $A$ is the von Neumann algebra $L^{\infty}\left(\mathbb{A}_{\mathbb{Q}}\right)$, and

$$
\varphi(f)=\int_{\mathbb{A}_{\mathbb{Q}}} f d \rho \text { for all } f \in A,
$$

where $\mathbb{A}_{\mathbb{Q}}$ is the Adele ring equipped with its unbounded measure $\rho$ on the $\sigma$-algebra of $\mathbb{A}_{\mathbb{Q}}$.

We now are interested in such free-moment $L$-functions in our setting. Let's fix the arithmetic $p$-prime probability $\operatorname{space}\left(\mathcal{A}, g_{p}\right)$ for a prime $p$.

Proposition 4.1. Let $\left(\mathcal{A}, g_{p}\right)$ be the arithmetic p-prime probability space. Then for any $f \in \mathcal{A}$, the free-moment $L$-function $L_{p}(f, s)$ satisfies

$$
L_{p}(f, s)=f(p)\left(\sum_{n=1}^{\infty} \frac{f(1)^{n-1}}{n^{s-1}}\right) .
$$

Proof. By the definition (4.1) of free-moment $L$-functions, if $f$ is a free random variable in $\left(\mathcal{A}, g_{p}\right)$, then

$$
L_{p}(f, s)=\sum_{n=1}^{\infty} \frac{g_{p}\left(f^{(n)}\right)}{n^{s}}=\sum_{n=1}^{\infty} \frac{n f(1)^{n-1} f(p)}{n^{s}}
$$

by $(3.5)$

$$
L_{p}(f, s)=\sum_{n=1}^{\infty} \frac{f(1)^{n-1} f(p)}{n^{s-1}}=f(p)\left(\sum_{n=1}^{\infty} \frac{f(1)^{n-1}}{n^{s}}\right) .
$$

By (4.2), we obtain the following corollary.

Corollary 4.2. Let $\left(\mathcal{A}, g_{p}\right)$ be the arithmetic p-prime probability space for a prime $p$, and let $f \in\left(\mathcal{A}, g_{p}\right)$. If $f$ is unital in the sense that: $f(1)=1$, then

$$
L_{p}(f, s)=f(p)(\zeta(s-1)),
$$

where $\zeta(s) \stackrel{\text { def }}{=} \sum_{n=1}^{\infty} \frac{1}{n^{s}}$ is the Riemann zeta-function.

Proof. Assume that a free random variable $f$ is unital in the sense that: $f(1)=$ 1. Then, by (4.2),

$$
L_{p}(f, s)=f(p)\left(\sum_{n=1}^{\infty} \frac{f(1)^{n-1}}{n^{s-1}}\right)=f(p)\left(\sum_{n=1}^{\infty} \frac{1}{n^{s-1}}\right)=f(p) \zeta(s-1),
$$

where $\zeta$ is the Riemann zeta-function, $\zeta(t)=\sum_{n=1}^{\infty} \frac{1}{n^{t}}$ for $t \in \mathbb{C}$.

Motivated by (4.3), one can get the following theorem. 
Theorem 4.3. Let $\left(\mathcal{A}, g_{p}\right)$ be the arithmetic p-prime probability space for a prime $p$, and let $f \in\left(\mathcal{A}, g_{p}\right)$, and $L_{p}(f, s)$, the corresponding free-moment $L$-function of $f$.

$$
\begin{aligned}
& \text { If }|f(1)| \leq 1, \text { then } L_{p}(f, s) \text { converges, whenever } \operatorname{Re}(s-1)>1 \text {. } \\
& \text { If }|f(1)|>1, \text { then } L_{p}(f, s) \text { diverges, for any } s \in \mathbb{C} \text {. }
\end{aligned}
$$

Proof. First, assume that $f(1)=0$, equivalently, $f \in K_{1}$ in $\mathcal{A}$. Then,

$$
L_{p}(f, s)=f(p)\left(\sum_{n=1}^{\infty} \frac{f(1)^{n-1}}{n^{s-1}}\right)=0,
$$

and hence it is convergent in $\mathbb{C}$, so it satisfies the statement (4.4).

Assume now that $f(1) \neq 0$, equivalently, $f \in K_{1}^{c}$ in $\mathcal{A}$. Observe now that

$$
\frac{f(1)^{n-1}}{n^{s-1}}=\frac{e^{\log f(1)^{n-1}}}{e^{\log n^{s-1}}}=\frac{e^{(n-1) \log f(1)}}{e^{(s-1) \log n}}=e^{(n-1) \log f(1)-(s-1) \log n}
$$

for all $n \in \mathbb{N}$.

Suppose that $0<|f(1)|<1$. Then the modulus of the power term of (4.6) satisfies

$$
\operatorname{Re}((n-1) \log f(1)-(s-1) \log n)<0,
$$

whenever $\operatorname{Re}(s-1)>1$. Therefore, the series

$$
\sum_{n=1}^{\infty} e^{(n-1) \log f(1)-(s-1) \log n}=\sum_{n=1}^{\infty} \frac{f(1)^{n-1}}{n^{s}}
$$

converges whenever $\operatorname{Re}(s-1)>1$.

Assume now that $|f(1)|=1$. Take $f$ as a unital element of $\mathcal{A}$, i.e., $f(1)=1$. Then, by (4.3),

$$
L_{p}(f, s)=f(p) \zeta(s-1),
$$

and hence, it converges whenever $\operatorname{Re}(s-1)>1$. More generally, if $|f(1)|=1$, then

$$
\left|L_{p}(f, s)\right|=|f(p)||\zeta(s-1)|,
$$

and hence, it converges, whenever $\operatorname{Re}(s-1)>1$.

Therefore, if $|f(1)| \leq 1$, then the corresponding free-moment $L_{p}(f, s)$ is convergent, whenever $\operatorname{Re}(s-1)>1$.

If $|f(1)|>1$, then

$$
\operatorname{Re}((n-1) \log f(1)-(s-1) \log n)>0,
$$

and, if $n \rightarrow \infty$,

$$
\operatorname{Re}((n-1) \log f(1)-(s-1) \log n) \rightarrow \infty
$$

for any fixed $s \in \mathbb{C}$. Therefore,

$$
\left|\lim _{n \rightarrow \infty} e^{(n-1) \log f(1)-(s-1) \log n}\right|=e^{\infty}=\infty,
$$

and hence, the series $\sum_{n=1}^{\infty} \frac{f(1)^{n-1}}{n^{s-1}}$ diverges in $\mathbb{C}$, whenever $|f(1)|>1$. 
The above theorem characterizes the convergence of our free-moment $L$ functions, i.e., the free-moment $L$-functions $L_{p}(f, s)$ converges, if $|f(1)| \leq 1$, and $\operatorname{Re}(s-1)>1$.

Define now arithmetic functions $a_{f}$ in $\mathcal{A}$ induced by other arithmetic functions $f$ of $\mathcal{A}$ by

$$
a_{f}(n) \stackrel{\text { def }}{=} f(1)^{n-1} \text { for all } n \in \mathbb{N} .
$$

By definition, one can define

$$
a_{a_{a_{a} a}} \quad, \text { etc, for } f \in \mathcal{A},
$$

inductively.

By definition, our free-moment $L$-function $L_{p}(f, s)$ on the arithmetic $p$-prime probability space $\left(\mathcal{A}, g_{p}\right)$ is determined by

$$
\begin{aligned}
L_{p}(f, s) & \stackrel{\text { def }}{=} \sum_{n=1}^{\infty} \frac{g_{p}\left(f^{n}\right)}{n^{s}}=\sum_{n=1}^{\infty} \frac{n f(1)^{n-1} f(p)}{n^{s}} \\
& =f(p)\left(\sum_{n=1}^{\infty} \frac{f(1)^{n-1}}{n^{s-1}}\right) \\
& =f(p)\left(\sum_{n=1}^{\infty} \frac{a_{f}(n)}{n^{s-1}}\right)
\end{aligned}
$$

for all $n \in \mathbb{N}, f \in \mathcal{A}$. So, we obtain the following theorem.

Theorem 4.4 (Also, see $[6])$. Let $\left(\mathcal{A}, g_{p}\right)$ be the arithmetic p-prime probability space. Then, for a free-moment $L$-function $L_{p}(f, s)$, there exists an arithmetic function $a_{f} \in \mathcal{A}$, such that (i) $a_{f}(n)=f(1)^{n-1}$ for all $n \in \mathbb{N}$, and (ii)

$$
L_{p}(f, s)=f(p)\left(L\left(a_{f}, s-1\right)\right)
$$

for all $f \in \mathcal{A}, s \in \mathbb{C}$, where $L\left(a_{f}, s-1\right)$ means the classical Dirichlet $L$-function induced by $a_{f} \in \mathcal{A}$, in the sense of Section 1.

The above relation (4.7) provides a characterization of free-moment $L$-functions in $\left(\mathcal{A}, g_{p}\right)$ in terms of classical $L$-functions: free-moment $L$-functions $L_{p}(f, s)$ of $f \in\left(\mathcal{A}, g_{p}\right)$ are understood as the products of the quantities $f(p)=$ $g_{p}(f)$, and classical Dirichlet $L$-functions induced by $a_{f} \in \mathcal{A}$.

Let $f, h$ be in $\left(\mathcal{A}, g_{p}\right)$, and let $L_{p}(f, s)$ and $L_{p}(h, s)$ be the corresponding free-moment $L$-functions. Then, by (4.7),

$$
L_{p}(f, s)=f(p)\left(L\left(a_{f}, s-1\right)\right),
$$

and

$$
L_{p}(h, s)=h(p)\left(L\left(a_{h}, s-1\right)\right) .
$$

Thus, one can get the following lemma. 
Lemma 4.5. (4.8) Let $L_{p}(f, s)$ and $L_{p}(h, s)$ be the free-moment $L$-functions of $f, h \in\left(\mathcal{A}, g_{p}\right)$. Then

$$
\left(L_{p}(f, s)\right)\left(L_{p}(h, s)\right)=(f(p) h(p))\left(L\left(a_{f} * a_{h}, s-1\right)\right) .
$$

(4.9) Let $L_{p}(f, s)$ and $L_{q}(h, s)$ be the free-moment $L$-functions of $f \in\left(\mathcal{A}, g_{p}\right)$ and $h \in\left(\mathcal{A}, g_{q}\right)$, where $p$ and $q$ are primes. Then

$$
\left(L_{p}(f, s)\right)\left(L_{q}(h, s)\right)=(f(p) h(q))\left(L\left(a_{f} * a_{h}, s-1\right)\right) .
$$

Proof. First, the formula (4.8) is trivial by (4.7). Note that, if $h_{1}$ and $h_{2}$ are arithmetic functions, then the corresponding classical $L$-functions satisfy that

$$
\left(L\left(h_{1}, s\right)\right)\left(L\left(h_{2}, s\right)\right)=L\left(h_{1} * h_{2}, s\right) .
$$

Therefore, even though $p$ and $q$ are primes, the corresponding free-moment $L$-functions are understood as products of certain quantities and classical Dirichlet $L$-functions. So,

$$
\begin{aligned}
\left(L_{p}(f, s)\right)\left(L_{q}(h, s)\right) & =\left(f(p) \sum_{n=1}^{\infty} \frac{a_{f}(n)}{n^{s-1}}\right)\left(h(p)\left(\sum_{n=1}^{\infty} \frac{a_{h}(n)}{n^{s-1}}\right)\right) \\
& =(f(p) h(p))\left(\sum_{n=1}^{\infty} \frac{\left(a_{f} * a_{h}\right)(n)}{n^{s-1}}\right) \\
& =(f(p) h(p)) L\left(a_{f} * a_{h}, s-1\right) .
\end{aligned}
$$

So, the formula (4.9) holds. As a special case of (4.9) (where $p=q$ ), the formula (4.8) holds, too.

By (4.8) and (4.9), we obtain the following theorem.

Theorem 4.6. Let $p_{1}, \ldots, p_{N}$ be primes which are not necessarily distinct, for $N \in \mathbb{N}$. Let $f_{j}$ be the free random variables of the arithmetic $p_{j}$-prime probability space $\left(\mathcal{A}, g_{p_{j}}\right)$, for $j=1, \ldots, N$. Then

$$
\prod_{j=1}^{N}\left(L_{p_{j}}\left(f_{j}, s\right)\right)=\left(\prod_{j=1}^{N} f_{j}\left(p_{j}\right)\right)\left(L\left(\underset{j=1}{N} a_{f_{j}}, s-1\right)\right) .
$$

By (4.4), (4.5), and (4.10), one obtains the following corollary.

Corollary 4.7. Let $p_{1}, \ldots, p_{N}$ be primes, for $N \in \mathbb{N}$. Let $f_{j}$ be the free random variables of the arithmetic $p_{j}$-prime probability space $\left(\mathcal{A}, g_{p_{j}}\right)$, and let $L_{p_{j}}\left(f_{j}, s\right)$ be the corresponding free-moment $L$-functions, for $j=1, \ldots, N$.

(4.11) If $\left|f_{j}(1)\right| \leq 1$ for all $j=1, \ldots, N$, then $\prod_{j=1}^{N}\left(L_{p_{j}}\left(f_{j}, s\right)\right)$ is convergent whenever $\operatorname{Re}(s-1)>1$.

(4.12) If $L_{p_{j}}\left(f_{j}, s\right)$ are convergent whenever $\operatorname{Re}(s-1)>1$ for all $j=1, \ldots, N$, then $\prod_{j=1}^{N}\left(L_{p_{j}}\left(f_{j}, s\right)\right)$ is convergent whenever $\operatorname{Re}(s-1)>1$. 
Recall now the identically-distributedness on $\mathcal{A}$ (in terms of primes). Two free random variables $f_{1}$ and $f_{2}$ are identically distributed in $\left(\mathcal{A}, g_{p}\right)$, if and only if

$$
f_{1}(1)=f_{2}(1), \text { and } f_{1}(p)=f_{2}(p) \text {, in } \mathbb{C},
$$

moreover, the quotient algebra $\mathfrak{A}_{p}=\mathcal{A} / \mathcal{R}_{p}$ is algebra-isomorphic to the algebra $\mathfrak{C}^{2}$.

So, under the identically distributedness on the arithmetic algebra $\mathcal{A}$, one can get the following theorem.

Theorem 4.8. Let $(\alpha, \lambda) \in \mathfrak{C}^{2}$. Then there exist free-moment L-functions of $f \in\left(\mathcal{A}, g_{p}\right)$ "for all primes $p$ " such that

$$
L_{p}(f, s)=\lambda\left(\sum_{n=1}^{\infty} \frac{\alpha^{n-1}}{n^{s-1}}\right) .
$$

Proof. Since the algebra $\mathfrak{C}^{2}$ is algebra-isomorphic to $\mathfrak{A}_{p}=\mathcal{A} / \mathcal{R}_{p}$, for every prime $p$, by (3.18) and (3.19). So, there exists at least one arithmetic function $f$ in $\left(\mathcal{A}, g_{p}\right)$, for each prime $p$, such that

$$
f(1)=\alpha, \text { and } g_{p}(f)=f(p)=\lambda,
$$

such that $[f]_{p} \in \mathfrak{A}_{p}$, for any prime $p$. Then, by the identically-distributedness $\mathcal{R}_{p}$ on $\left(\mathcal{A}, g_{p}\right)$, for any $h \in[f]_{p}$,

$$
L_{p}(h, s)=h(p)\left(\sum_{n=1}^{\infty} \frac{h(1)^{n-1}}{n^{s-1}}\right)
$$

by $(4.7)$

$$
L_{p}(h, s)=\lambda\left(\sum_{n=1}^{\infty} \frac{\alpha^{n-1}}{n^{s-1}}\right) .
$$

Notice that the above theorem holds for all primes.

Define a set

$$
\mathcal{L}_{p} \stackrel{\text { def }}{=}\left\{L_{p}(f, s): f \in\left(\mathcal{A}, g_{p}\right)\right\}
$$

for all primes $p$. Then this set $\mathcal{L}_{p}$ becomes an algebra under the usual functional addition $(+)$ and the usual functional multiplication $(\cdot)$.

Define a morphism $\psi_{p}$ from the arithmetic algebra $\mathcal{A}$ into $\mathcal{L}_{p}$ by

$$
\psi_{p}(f) \stackrel{\text { def }}{=} L_{p}(f, s),
$$

then it is a well-defined algebra-epimorphism, satisfying

$$
\psi_{p}\left(f_{1} * f_{2}\right)=\left(\psi_{p}\left(f_{1}\right)\right)\left(\psi_{p}\left(f_{2}\right)\right)
$$

for all $f_{1}, f_{2} \in\left(\mathcal{A}, g_{p}\right)$, for each prime $p$. So, one can verify the following isomorphism theorem. 
Theorem 4.9. Let $\mathfrak{A}_{p}=\mathcal{A} / \mathcal{R}_{p}$, and assume $\Psi_{p}=\psi_{p} / \mathcal{R}_{p}$ is the quotient map from $\mathfrak{A}_{p}$ to $\mathcal{L}_{p}$, where $\mathcal{L}_{p}$ is an algebra (4.14), and $\psi_{p}$ is in the sense of (4.15). Then $\Psi_{p}$ is an algebra-isomorphism. Equivalently,

$$
\mathfrak{A}_{p} \stackrel{\text { Algebra }}{=} \mathcal{L}_{p} .
$$

Proof. Let $\mathfrak{A}_{p}=\mathcal{A} / \mathcal{R}_{p}$, and let $\mathcal{L}_{p}$ be in the sense of (4.14). Since $\mathcal{A}$ is epimorphic to $\mathcal{L}_{p}$, the quotient map $\Psi_{p}$ is an algebra-homomorphism from $\mathfrak{A}_{p}$ to $\mathcal{L}_{p}$. Since each $L_{p}(f, s)$ satisfies

$$
L_{p}(f, s)=f(p) \sum_{n=1}^{\infty} \frac{f(1)^{n-1}}{n^{2}},
$$

if $(f(1), f(p))=(\alpha, \lambda)$ in $\mathbb{C}^{2}$, then

$$
L_{p}(h, s)=\lambda \sum_{n=1}^{\infty} \frac{\alpha^{n-1}}{n^{s-1}}
$$

for all $h \in[f]_{p}$. Thus, under quotient, $f$ is uniquely determined up to $\mathcal{R}_{p}$, i.e., $\Psi_{p}$ is injective.

So, $\Psi_{p}: \mathfrak{A}_{p} \rightarrow \mathcal{L}_{p}$ is an algebra-isomorphism, equivalently, two algebras $\mathfrak{A}_{p}$ and $\mathcal{L}_{p}$ are isomorphic.

By (4.16), we obtain a following isomorphism theorem, too.

Corollary 4.10. Let $\mathcal{L}_{p}$ be an algebra in the sense of (4.14), for primes $p$. Then

$$
\mathcal{L}_{p} \stackrel{\text { Algebra }}{=} \mathfrak{C}^{2} \text { for all primes } p,
$$

where $\mathfrak{C}^{2}$ is an algebra in the sense of (3.18).

Proof. The proof is done by (3.19).

So, the algebra $\mathcal{L}_{p}$ of all $g_{p}$-free-moment $L$-functions induced by $\mathcal{A}$ is classified by $\mathfrak{C}^{2}$.

\section{References}

[1] T. M. Apostol, Modular Functions and Dirichilet Series in Number Theory, SpringerVerlag, 1990

[2] D. Bump, Automorphic Forms and Representations, Cambridge Studies in Adv. Math., 55, Cambridge Univ. Press., 1996.

[3] I. Cho, Direct producted $W^{*}$-probability spaces and corresponding amalgamated free stochastic integration, Bull. Korean Math. Soc. 44 (2007), no. 1, 131-150.

[4] - Group freeness and certain amalgamated freeness, J. Korean Math. Soc. 45 (2008), no. 3, 597-609.

[5] - On dynamical systems induced by the Adele ring, Global J. Sci. Frontier Research (2015), To appear.

[6] - Free distributional data of arithmetic functions and corresponding generating functions determined by gaps of primes, Compl. Anal. Opre. Theo. 8 (2014), no. 2, $537-570$. 
[7] _ Dynamical systems on arithmatic functions determined by primes, Banach J. Math. Anal. 9 (2015), no. 9, 173-215.

[8] I. Cho and T. Gillespie, Arithmetic functions and free moments of certain free random variables, Preprint, 2012

[9] I. Cho and P. E. T. Jorgensen, $C^{*}$-subalgebras generated by partial isometries, J. Math. Phy., DOI://10.1063/1.3056588, 2009.

[10] - Free probability induced by electric resistance networks on energy Hilbert spaces, Opuscula Math. 31 (2011), no. 4, 549-598.

[11] H. Davenport, Multiplicative Number Theory, 3-rd Ed., Grad. Texts in Math., vol. 74, Springer-Verlag, 2000

[12] T. Gillespie, Superposition of zeroes of automorphic L-functions and functoriality, Ph.D. Thesis., The University of Iowa., 2011.

[13] - Prime number theorems for Rankin-Selberg L-functions over number fields, Sci. China Math. 54 (2011), no. 1, 35-46.

[14] A. J. Hildebrand, Introduction to Analytic Number Theory, Lecture Notes, availble at http://www.math.uiuc.edu/ ${ }^{\text {hilderbr/ant, } 2006 .}$

[15] F. Radulescu, Random matrices, amalgamated free products and subfactors of the $C^{*}$ algebra of a free group of nonsingular index, Invent. Math. 115 (1994), no. 2, 347-389.

[16] R. Speicher, Combinatorial theory of the free product with amalgamation and operatorvalued free probability theory, Mem. Amer. Math. Soc. 132 (1998), no. 627, x+88 pp.

[17] V. S. Vladimirov, I. V. Volovich, and E. I. Zelenov, p-Adic Analysis and Mathematical Physics, Ser. Soviet \& East European Math., vol 1, World Scientific, 1994.

[18] D. Voiculescu, K. Dykemma, and A. Nica, Free Random Variables, CRM Monograph Series, vol 1., 1992.

Department of Mathematics

St. Ambrose University

421 Ambrose Hall, $518 \mathrm{~W}$. Locust St.

DAVENPORT, IOWA, 52803, USA

E-mail address: choilwoo@sau.edu 